Bangladesh J. Plant Taxon. 21(1): 27-32, 2014 (June)

(C) 2014 Bangladesh Association of Plant Taxonomists

\title{
A NEW VARIETY AND TWO NEW RECORDS OF ELATOSTEMA J. R. FORSTER \& G. FORSTER (URTICACEAE) FROM VIETNAM
}

\author{
Yun Lin, Lin Dong DUAN ${ }^{1}$ AND HaI Yan Bi ${ }^{2}$ \\ Hunan Food and Drug Vocational College, Changsha 410014, P. R. China
}

Keywords: Elatostema; New record; New variety; Urticaceae; Vietnam.

\begin{abstract}
Based on examination of specimens of Elatostema J. R. Forster \& G. Forster (Urticaceae) from Vietnam, Elatostema albopilosum W.T. Wang var. vicinum L. D. Duan \& Y. Lin, a new variety collected from Bac Can Province and Tuyen Quang Province, Vietnam, is described and photographed. This new variety has glabrous stems, glabrous leaves and glabrous peduncles which differs from var. albopilosum that having sparsely puberulent leaf blade on abaxial surface along veins and sparsely strigillose leaf blade on adaxial surface, and short pilose peduncles. E. integrifolium (D. Don) Wedd. and E. pseudodissectum W. T. Wang, two species previously known from Vietnamese adjacent countries, are newly recorded from Ninh Binh Province and Tam Dao National Park, Vietnam, respectively. The vouchers are kept in the Herbarium (PE) of Institute of Botany, Chinese Academy of Sciences, Beijing, China, and their descriptions and photographs are also provided.
\end{abstract}

\section{Introduction}

The genus Elatostema J. R. Forster \& G. Forster is the largest genus in the family Urticaceae, consists of c. 500 species and is distributed in tropical and subtropical regions of Africa, Asia and Oceania, 30 species of which are found in Vietnam (Fu et al., 2013; Gagnepain, 1929; Ho, 2003; Lin, 2008; Lin et al., 2003, 2011; Wang, 1995, 2012). Based on examination of Elatostema specimens housed at the Herbarium (PE) of Institute of Botany, Chinese Academy of Sciences, Beijing, China, and after thoroughly consulting the literature (Gagnepain, 1929; Lin, 2008; Bi et al., 2011; Lin et al., 2003, 2011; Wang, 1980, 1995, 2012; Duan and Lin, 2013; Wei et al., 2013), a new variety was found from Vietnam, and two species previously known from Vietnamese adjacent countries, were discovered to be new to Vietnam, and were reported here.

1. Elatostema albopilosum W. T. Wang var. vicinum L. D. Duan \& Y. Lin, var. nov. (Figs 1, 2).

Diagnosis: The new variety Elatostema albopilosum W. T. Wang var. vicinum L. D. Duan \& Y. Lin is morphologically similar to E. albopilosum W. T. Wang var. albopilosum, but differs in glabrous stems, glabrous leaves and glabrous peduncles; the latter pilose stems, sparsely puberulent leaf blade on abaxial surface along veins, sparsely strigillose leaf blade on adaxial surface, and short pilose peduncles.

Type: Vietnam. Tuyen Quang Province, Na hang, 400 m a.s.l., 28 October 1998, H. N. Qin, H. Zhu, M. L. Zhang \& T. G. Gao 319 (Holotype: PE). Bac Can Province, Cho Don District, Xuan Lac Commune, 105'32'95'”E, 22 ${ }^{\circ} 21^{\prime}$ '91'”N, 200 m a.s.l., 27 October 2004, L. Q. Li, N. T. Hiep, Z. Y. Zhang, X. C. Zhang, T. G. Gao, Z. T. Wang, N. S. Khang \& N. X. Tam 0432 (Paratype: PE).

${ }^{1}$ Shaoyong University, Shaoyang 422004, P. R. China

${ }^{1}$ Beijing Museum of Natural History, Beijing 100050, P. R. China. Corresponding author. Email: rubybi@126.com 


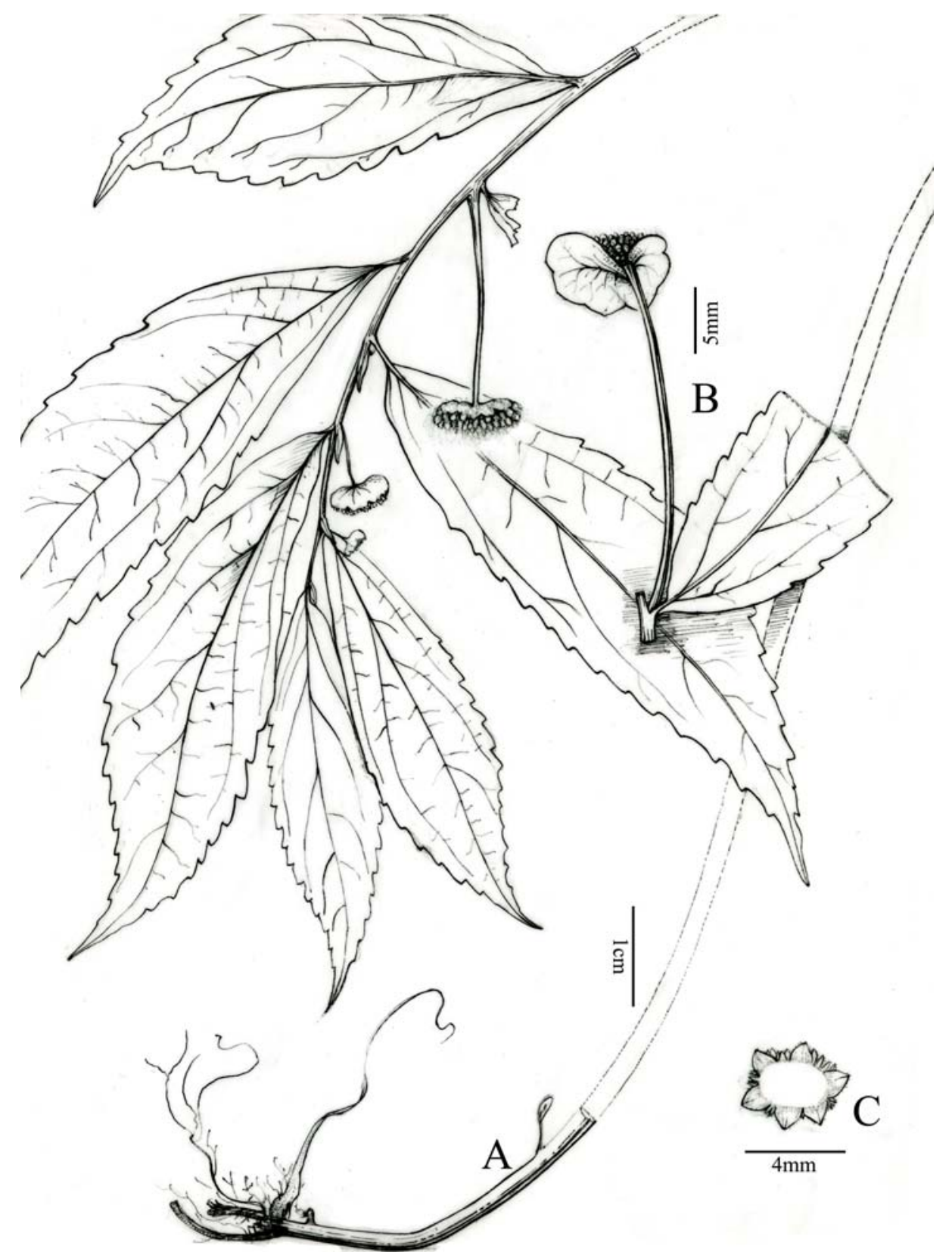

Fig. 1. Elatostema albopilosum W. T. Wang var. vicinum L. D. Duan \& Y. Lin, var. nov. A) male habit (H. $N$. Qin et al. 319, PE), B) staminate inflorescence (L. Q. Li et al. 0432, PE), C) pistillate inflorescence, dorsal view. (L. Q. Li et al. 0432, PE). 


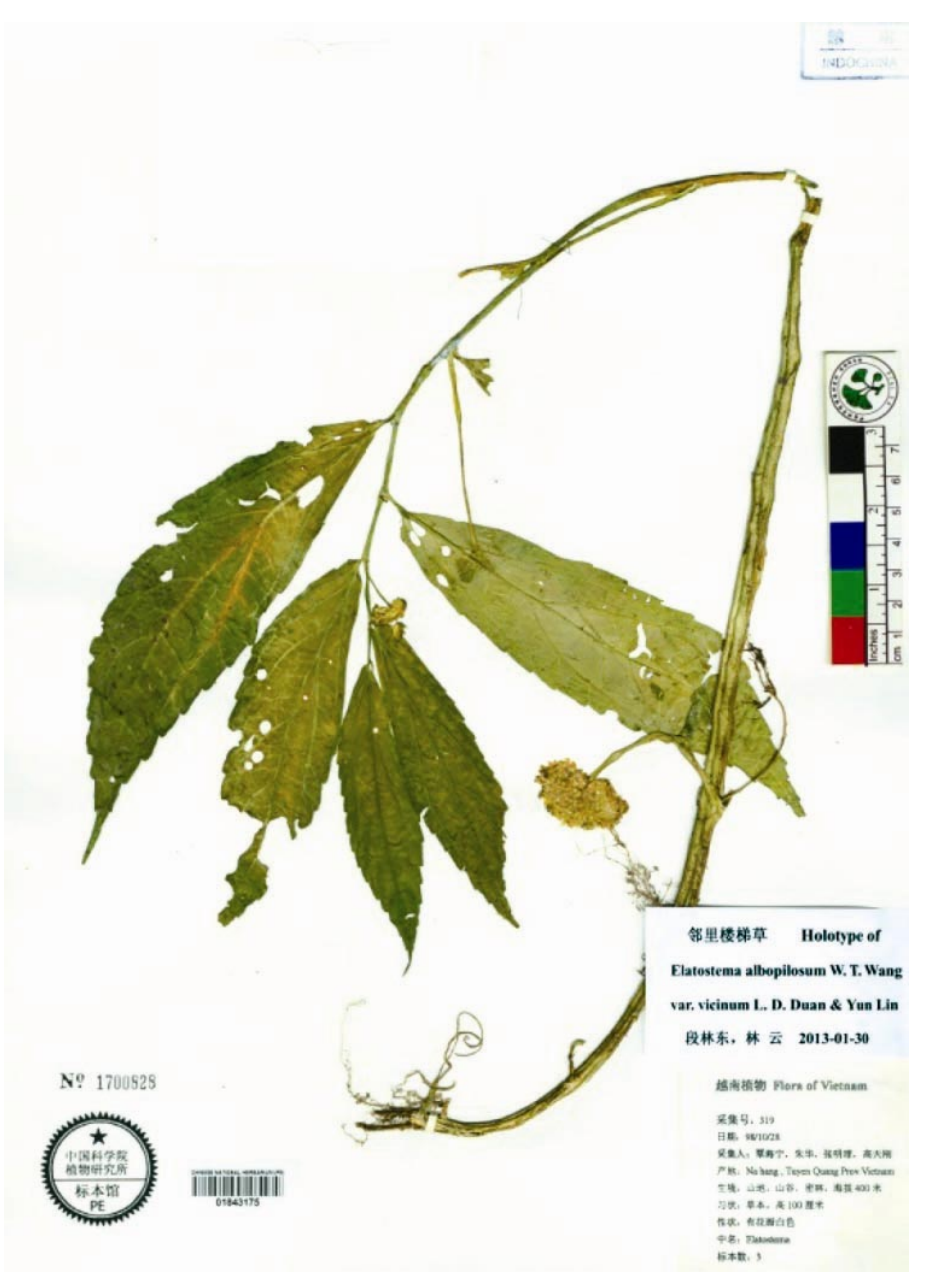

Fig. 2. Holotype of Elatostema albopilosum W. T. Wang var. vicinum L. D. Duan \& Y. Lin (from H. N. Qin et al. 319, PE)

2. Elatostema integrifolium (D. Don) Wedd. in DC., Prodr. 16(1): 179 (1869). Procris integrifolia D. Don, Prodr. Fl. Nepal. : 61 (1825).

(Fig. 3).

Type: Nepal. Precise locality not known, Wallich s. n. (? K).

Perennial herbs or subshrubs, monoecious or dioecious, $60-200 \mathrm{~cm}$ tall. Stems erect, branched, glabrous. Leaves alternate, glabrous; nanophylls absent; stipules narrowly lanceolate, 6$10 \times 1.5-2.0 \mathrm{~mm}$, with cystoliths; petiole 1.5-6.0 (-10) mm long; leaf blade obliquely elliptic or obliquely oblanceolate, 5-19 × 2-6 cm, papery or herbaceous, major basal lateral veins asymmetric, 1 basal, the other arising above base, cystoliths conspicuous, dense, 0.2-0.4 (-0.6) mm long; base obliquely cuneate, margin entire or c. 2-crenate, apex acuminate or caudate. Male inflorescence solitary or in pairs, simple, $10-18 \times 6-12 \mathrm{~mm}$, sessile; receptacle $4-6 \mathrm{~mm}$ in diam.; bracts 4 , triangular, equal, connate, c. $1 \mathrm{~mm}$ long; bracteoles cymbiform, c. $1.2 \mathrm{~mm}$ long. Female inflorescence solitary or in pairs, 5-8 $\mathrm{mm}$ in diam.; peduncle $0-1 \mathrm{~mm}$ long; receptacle very small; bracts 20-30, triangular, nearly equal, $0.8 \mathrm{~mm}$ long; bracteoles narrowly linear, $0.8-1.2 \mathrm{~mm}$ long. 
Male flowers 4-merous. Achenes ellipsoid, $0.7 \mathrm{~mm}$ long, 8-ribbed.

Phenology: Flowering from February to May.

Distribution: Bhutan, China, India, Indonesia, Myanmar, Nepal and Thailand. New record to Vietnam.

Habitat: The species grows in valley forests, streamsides at altitudes of $460-760 \mathrm{~m}$ in Vietnam and 900-1600 $\mathrm{m}$ in other countries.

Specimens examined: Vietnam. Ninh Binh Province: Bong, Biology Station, 460-760 m a.s.l., 10.2.1965, Sino-Vietnam Exped. 2332 (PE).

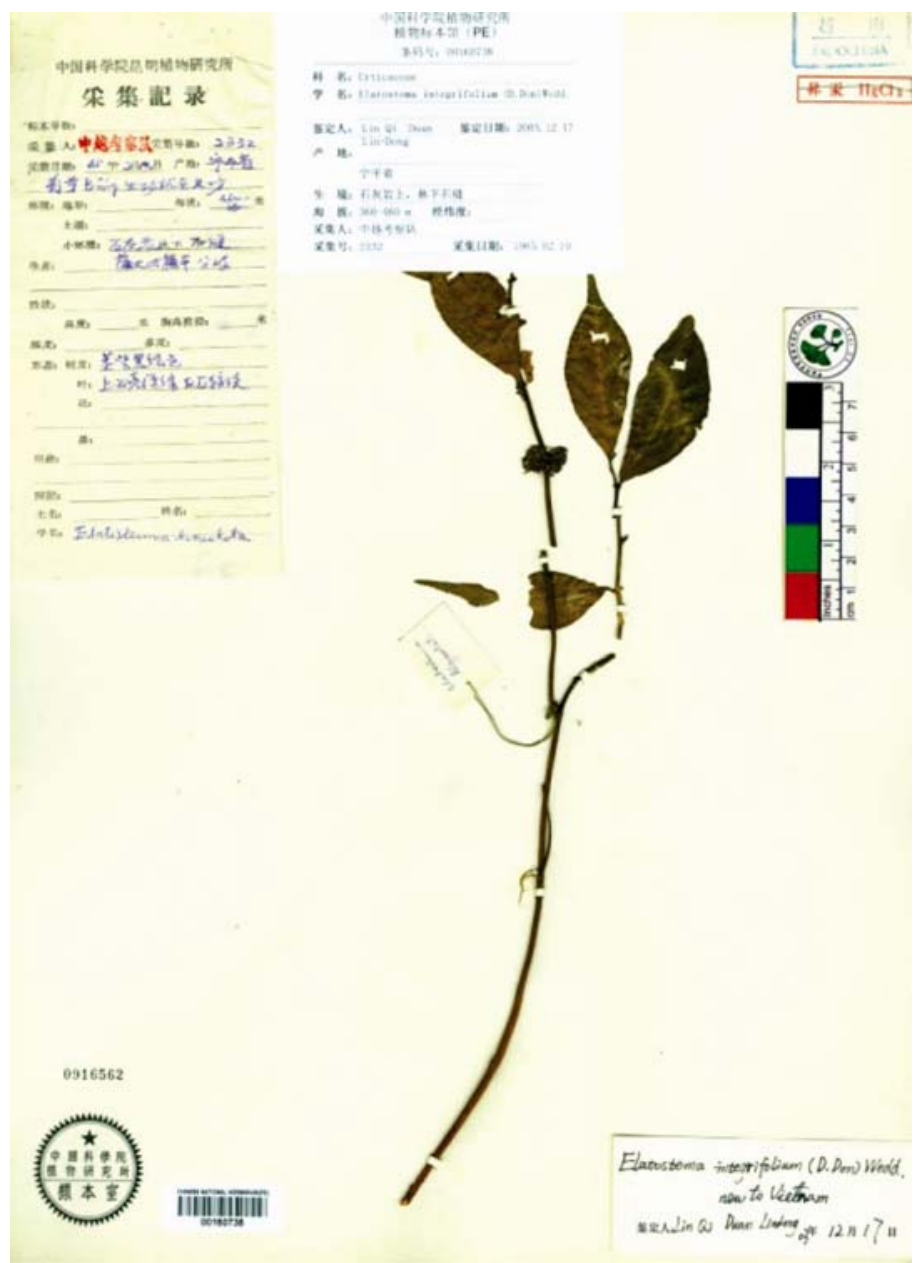

Fig. 3. Elatostema integrifolium (D. Don) Wedd. (from Sino-Vietnam Exped. 2332, PE).

Note: This newly recorded species is similar to E. laevissimum W.T. Wang (Wang, 1980) in Vietnam, but differing in stipules narrowly lanceolate, 6-10 $\times 1.5-2.0 \mathrm{~mm}$, with cystoliths, leaves glabrous, male inflorescence 10-18 $\times$ 6-12 mm, female inflorescence 5-8 mm in diam., the latter with stipules narrowly triangular, $2-5 \times 0.8-1.0 \mathrm{~mm}$, without cystoliths, leaf blade sparsely 
strigillose on adaxial surface, male inflorescence 3-4 $\mathrm{mm}$ in diam. and female inflorescence 1.5$2.5 \mathrm{~mm}$ in diam.

3. Elatostema pseudodissectum W. T. Wang, Bull. Bot.. Lab. N.-E. For. Inst. 7(7): 55 (1980). (Fig. 4).

Type: China. Guangxi: Baise, 15 August 1928, R. C. Ching 7427 (Holotype: PE!).

Perennial herbs, monoecious, 20-40 cm tall, glabrous. Stems ascending or erect, branched. Leaves alternate; nanophylls absent; stipules narrowly triangular, 0.7-1.5 $\times$ 0.2-0.4 mm, without cystoliths; petiole 1-3 mm long; leaf blade obliquely ovate or elliptic-ovate, $4-11 \times 1.5-4.0 \mathrm{~cm}$, membranous or herbaceous, major basal lateral veins both arising at base of leaf blade, cystoliths conspicuous, dense, 0.2-0.6 mm long; base obliquely cuneate, apex acuminate or cuspidate, margin denticulate. Male inflorescence solitary, simple, 4-7 mm in diam.; peduncle 12-40 mm long; receptacle small; bracts 3-4, connate, equal, broadly ovate. Female inflorescence solitary, 30flowered or more, 3-6 mm in diam., sessile; receptacle 3-5 mm in diam.; bracts 11-20, narrowly ovate, nearly equal, c. $1.2 \mathrm{~mm}$ long; bracteoles spathulate-linear, 0.3-0.5 mm long. Male flowers 4-merous. Achenes ellipsoid, c. $0.6 \mathrm{~mm}$ long, smooth.

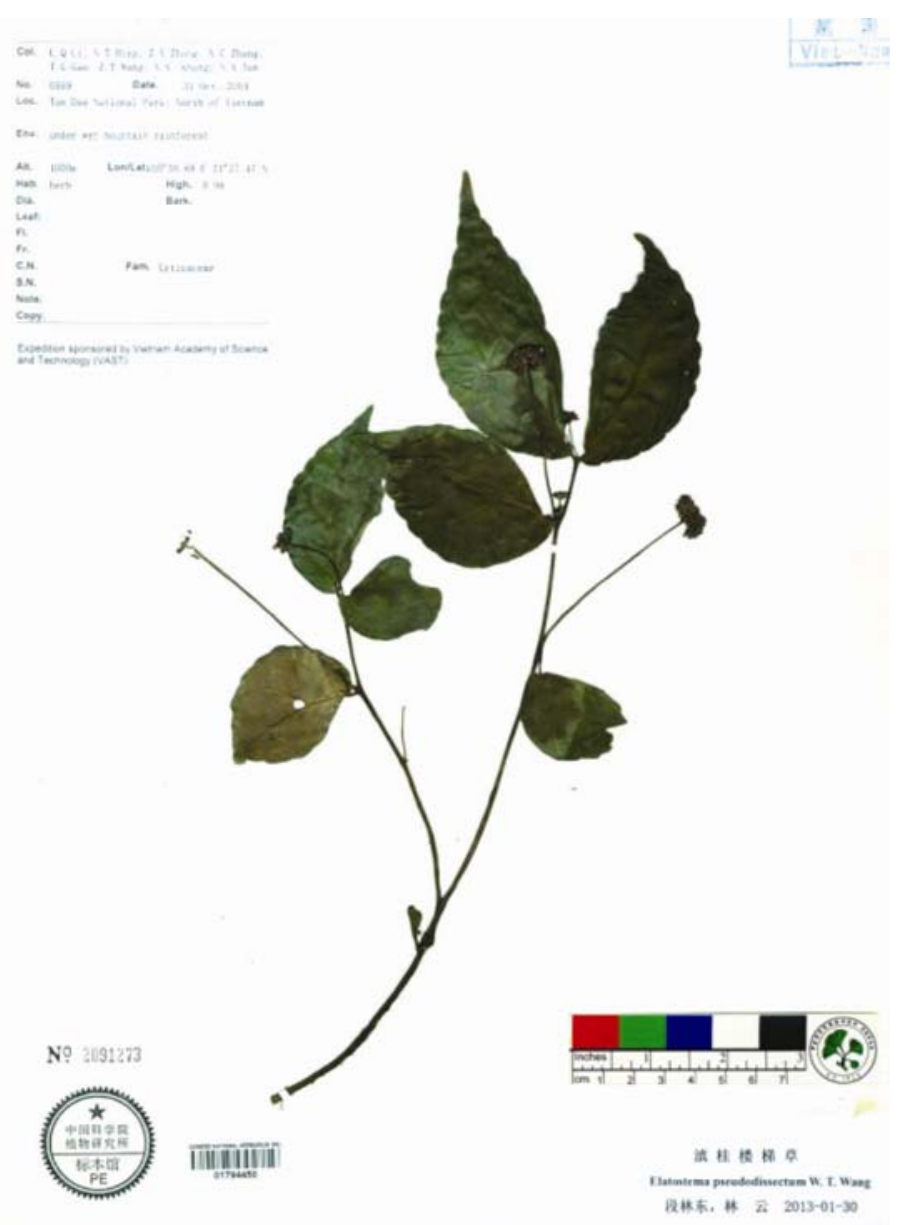

Fig. 4. Elatostema pseudodissectum W. T. Wang (from L. Q. Li et al. 559, PE). 
Phenology: Flowering from September to October.

Distribution: South-west China. New record to Vietnam.

Habitat: This species grows in valley wet forests, streamsides at altitudes of $1000 \mathrm{~m}$ in Vietnam and 1100-2200 $\mathrm{m}$ in China.

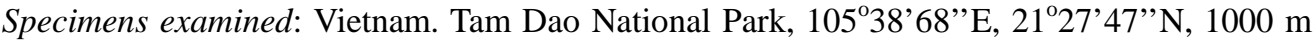
a.s.l., 31.10.2004, L. Q. Li, N. T. Hiep, Z. Y. Zhang, X. C. Zhang, T. G. Gao, Z. T. Wang, N. S. Khang \& N. X. Tam 559 (PE). Precise locality not known, Ke Can, 150 m a.s.l., 5.1.1965, SinoVietnam Exped. 1750 (PE).

Note: This newly recorded species is similar to E. dissectum Wedd. (Weddell, 1856) in Vietnam, differing in stipules narrowly triangular, 0.7-1.5 $\times 0.2-0.4 \mathrm{~mm}$, without cystoliths, leaf blade obliquely ovate or elliptic-ovate, male inflorescence 4-7 $\mathrm{mm}$ in diam., the latter having stipules narrowly linear or subulate, $3-5 \times 0.1-0.3 \mathrm{~mm}$, with cystoliths, leaf blade obliquely oblong or obliquely oblong-lanceolate and male inflorescence 10-12 × 8-10 mm.

\section{Acknowledgments}

Thanks are due to the Curator of herbarium (PE) for permission to examine specimens. This work was supported by the Project of the Education Department in Hunan Province (11A109).

\section{References}

Bi, H.Y., Yang, Z.R. and Lin, Q. 2011. New taxa of Elatostema (Urticaceae) from Thailand and India. Bangladesh J. Plant Taxon. 18(2) : 149-152.

Duan, L.D. and Lin, Y. 2013. Elatostema bioppositum (Urticaceae), a new species from Guangxi, China. Bangladesh J. Plant Taxon. 20(2) : 179-183.

Fu, L.F., Do, V.T., Wen, F., Liu, S.Y. and Wei, Y.G. 2013. New records of Elatostema and Pellionia (Urticaceae) from Vietnam. Guihaia 33(6): 801-803.

Gagnepain, F. 1929. Pellionia Gaudich. Flore Generale de L’Indo-Chine 5(9): 892-921.

Ho, P.H. 2003. An Illustrated Flora of Vietnam. Vol. 2. NXB Tre Press, Ho Chi Minh, pp. 589-593.

Lin, Q. 2008. A revision of Elatostema section Weddelia series Salvinioida (Urticaceae). Bot. J. Linn. Soc. 158(1): 62-66.

Lin, Q., Friis, I. and Wilmot-Dear, C.M. 2003. Elatostema (Urticaceae). In: Wu, Z.Y. and Raven, P.H. (Eds), Flora of China. Vol. 5. Science Press, Beijing, and Missouri Botanical Garden Press, St. Louis, USA, pp. 127-163.

Lin, Q., Yang, Z.R., Duan, L.D. and Gao, T.G. 2011. Miscellaneous taxonomic notes on Elatostema (Urticaceae) from China and its adjacent area. Nordic J. Bot. 29(5): 590-597.

Wang, W.T. 1980. Classificatio specierum sinicarum Elatostematis (Urticaceae). Bull. Bot. Lab. N.-E. For. Inst. 7(7): 1-96.

Wang, W.T. 1995. Elatostema J.R. Forster \& G. Forster. In: Wang, W.T. and Chen, C.J. (Eds), Flora Reipublicae Popularis Sinicae, Tomus 23, No. 2. Science Press, Beijing, pp. 187-317.

Wang, W.T. 2012. Nova Classificatio Specierum Sinicarum Elatostematis (Urticaceae) In: Fu, D.Z. (Ed.), Paper Collection of W.T. Wang. Vol. 2. Higher Education Press, Beijing, pp. 1061-1178.

Weddell, H.A. 1856. Monograhpie de la famille des Urticées. Archives du Muséum D’Histoire Naturelle 9: $1-332$.

Wei, Y.G., Wen, F., Fu, L.F. and Wang, W.T. 2013. Three new species of Elatostema J.R. Forst. \& G. Forst. (Urticaceae) in limestone caves from Guangxi and Guizhou, China. Bangladesh J. Plant Taxon. 20(1): $1-8$. 